\section{Imaging Parameters Used in the Sequence Tables}

The purpose of this appendix is to elaborate on some of the parameters used in the sequence tables included in the individual units. This may help the reader to understand those imaging parameters when it is necessary to translate them into parameters for a particular scanner, which will vary depending upon the manufacturer. The reader is also referred to UNIT A7.4 because some additional explanation is provided there.

Cine gradient echo sequence: In this sequence, the repeat time $\left(T_{\mathrm{R}}\right)$ listed is the effective repeat time-i.e., temporal resolution.

Delay time $\left(\boldsymbol{T}_{\mathrm{D}}\right)$ : Sometimes this is referred to as the delay time after the R wave.

Display matrix $\left(\boldsymbol{D}_{\mathrm{x}}, \boldsymbol{D}_{\mathrm{y}}\right)$ : This corresponds to the size of an image. The reader should not confuse this with "number of data points collected."

Echo train length (ETL): We define this as the number of $k$-space lines collected after the $\pi / 2$-pulse in fast spin echo sequences. This parameter belongs to a subgroup of the "number of lines per segment" collected in $k$-space.

Fast spin echo: This sequence is also called a turbo spin echo sequence.

Flip angle (FA): Generally speaking, the flip angle is the angle whose value is displayed on the MR console. For some spin echo sequences, the system displays the angle of the refocusing pulse instead of the $\pi / 2$ pulse.

Multi-phase: This is the terminology used by GE; it is similar to the number of repetitions, but is usually used when the sequence is a perfusion or MR angiography scan and it is desired to continually collect data after the injection of a contrast agent.

Number of acquisitions $\left(N_{\text {acq }}\right)$ : This is the number of times that a sequence is repeated, but the same number of images is produced whether $N_{\text {acq }}=1$ or some larger integer. Increasing the number of acquisitions increases the signal-to-noise ratio by the square root of the number of acquisitions. One should not confuse this parameter with number of repetitions. When this parameter is less than one, it means partial Fourier is used in the sequence. To GE users, this parameter is equivalent to the "number of excitations" parameter which is called NEX. In Marconi's system, this term is referred to as the "number of signal averages."

Number of acquisitions on a GE system: Unfortunately, GE uses this term to refer to the number of repetitions of a scan when there is insufficient time for short $T_{\mathrm{R}}$ sequences to collect all the slices of interest. For example, if only 10 slices can be run with a given $T_{\mathrm{R}}$ and $T_{\mathrm{E}}$ and 30 slices are required to cover the region of interest (ROI), GE scanners will run this scan three times but appropriately shift the slices to ensure the full coverage of the ROI.

Number of cardiac phases: This is the number of phases (or frames or images) of one slice that can be obtained in one heartbeat. This number is usually calculated as (R-to-R interval) multiplied by $85 \%$, divided by $T_{\mathrm{R}}$.

Number of data points collected $\left(N_{\mathrm{x}}, N_{\mathrm{y}}\right)$ : This is the acquisition matrix and it is the one that the reader needs to choose on the system before running the scan. $N_{\mathrm{x}}$ is the number of points collected in the read direction and $N_{\mathrm{y}}$ is the number of phase-encoding steps. If
Imaging

Parameters Used in the Sequence

Tables

A2.1

Supplement 12

Current Protocols in Magnetic Resonance Imaging (2004) A2.1-A2.2

Copyright $\odot 2004$ by John Wiley \& Sons, Inc. 
the sequence swaps the read and phase encoding directions, then $N_{\mathrm{x}}$ will be the number of phase-encoding steps and $N_{\mathrm{y}}$ will be the sampling points in the read direction. This setting is not the same as the "display matrix" (see above) which represents the number of pixels used to display an image.

Number of excitations (NEX): See $N_{\text {acq }}$ above.

Number of lines per segment: This is the number of $k$-space lines that can be collected in one $T_{\mathrm{R}}$ for a 2-D slice or a 3-D slab.

Number of repetitions: This is the number of times that a sequence is repeated. The number of images is proportional to this setting. Increasing the number of repetitions will increase the scan time but will not increase the signal-to-noise ratio. In Siemens' terminology, this is called the "number of measures."

Number of slices: This is also referred to as the "number of partition encoding steps" if a 3-D sequence is performed.

Read direction: This is also called the frequency direction.

Receiver bandwidth (RBW): This value has been quoted as plus or minus a frequency (e.g., $\pm 12 \mathrm{kHz}$ ) for GE's scanners. This means that, in GE's scanners, the sampling time along the readout direction is $N_{\mathrm{x}} /(2 \times \mid$ receiver bandwidth $\mid)$. In Siemens Sonota systems, in order to change the echo time, sometimes it is necessary to change the receiver bandwidth.

Rectangular fields of view: We usually give the true values of "fields of view $\left(\mathrm{FOV}_{\mathrm{x}}\right.$, $\mathrm{FOV}_{\mathrm{y}}$ )" in this series. The aspect ratio for a rectangular field of view is equal to $\mathrm{FOV}_{\mathrm{y}} /$ $\mathrm{FOV}_{\mathrm{x}}$.

Slab thickness: This is the "number of slices" multiplied by "slice thickness" $(\Delta z)$.

Swap read and phase encoding: In this series, we assume that the default setting of the read direction for transverse images is left-right. Thus, in order to avoid ghosting from the eyes in transverse images in the brain, one would usually swap read and phase-encoding directions. (However, this is not done for an echo planar sequence.) The default setting of the read direction in the other two orientations is superior-inferior; however, readers are strongly advised to check with the technicians at their own site for this setting.

Zerofill interpolation process (ZIP): This is an image reconstruction process. An image can be zero-filled and interpolated to any matrix size for display. For example, if an image is collected at an acquisition matrix $\left(N_{\mathrm{x}}, N_{\mathrm{y}}\right)$ of 256 by 256, then "ZIP 512" means that this image now has a display matrix $\left(D_{\mathrm{x}}, D_{\mathrm{y}}\right)$ of 512 by 512 and thus the in-plane resolution of this image is doubled. Under the same explanation, "ZIP 2" means that the throughplane resolution of an image is doubled. This process is accomplished by filling $k$-space with zeros out to the desired value and then Fourier-transforming the data. 